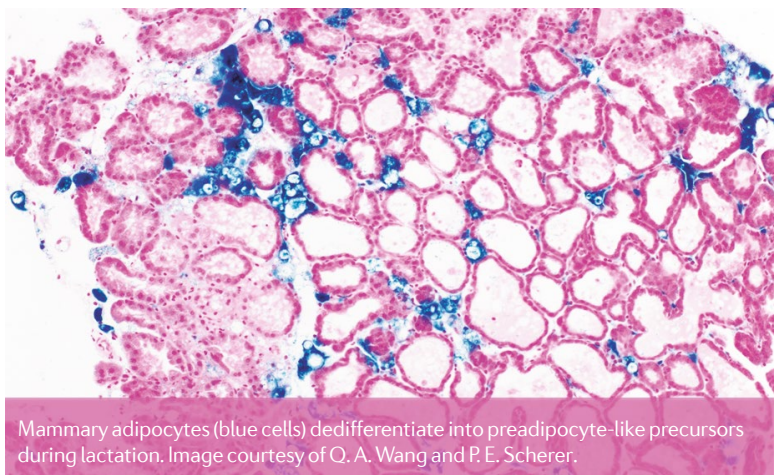

ADIPOSE TISSUE

\section{Plasticity of fat cells}

The origin of adipocytes in the mammary gland and mammary alveolar structures during pregnancy and lactation has been a matter of debate for some time. Various hypotheses have been put forward, including that mammary adipocytes transdifferentiate into mammary alveolar cells. In a new paper, Philipp Scherer and colleagues show that white adipocytes in the mammary gland dedifferentiate into precursor cells during pregnancy and lactation and then redifferentiate after weaning.

The researchers interbred three transgenic strains of mouse (including one expressing the LacZ reporter gene) to create a doxycycline-based tet-responsive Cre-loxP system, named AdipoChaser-LacZ. The mature adipocytes of these mice are permanently labelled, allowing their fate to be monitored over time. AdipoChaser-LacZ mice were bred, and mammary gland samples were collected during pregnancy, lactation and involution.

Scherer and colleagues found that none of the developing mammary gland structures stained positive for LacZ, which shows that these cells are not transdifferentiated from mature adipocytes. Importantly, they were also able to show that LacZ-positive adipocytes gradually lost their lipid droplets during lactation, so that they morphologically resembled fibroblasts. By the tenth day of lactation, these cells still stained positive for LacZ but were reduced in size and contained no lipid droplets. "The current text book suggests that fat cells only have three options: they can get bigger as we gain weight; they can get smaller as we lose weight; or they can die and cause inflammation," says Scherer. "This paper shows for the first time that fat cells have an additional option: they can dedifferentiate." After weaning, Scherer and co-workers found that adipocytes regenerated. These adipocytes were all positive for LacZ, indicating that they were derived from the original pool of mature adipocytes. Further experiments showed that these adipocytes dedifferentiated and redifferentiated through a second pregnancy.

"This dedifferentiation may also occur during weight loss and explain why fat cells 'come back' so easily," concludes Scherer. "These cells stick with us for much longer than anticipated, and may disguise themselves as fibroblasts that are ready to come right back and turn into fat cells."

Claire Greenhill

ORIGINAL ARTICLE Wang, Q. A. et al. Reversible de-differentiation of mature white adipocytes in preadipoctye-like precursors during lactation. Cell Metab. https://doi. org/10.1016/j.cmet.2018.05.022 (2018)

MICROBIOTA

\title{
New role for indoleamine 2,3-dioxygenase
}

Indoleamine 2,3-dioxygenase (IDO) is known to be involved in immune function and catalyses the degradation of tryptophan. Now, new research published in Nature Medicine demonstrates that this enzyme also has a role in metabolic health, via effects on the gut microbiota.

Increased levels of IDO have been observed in patients with cardiovascular disease and seem to be predictive of poorer outcomes. However, the mechanisms underlying this association are unclear. "As obesity is a major contributor to cardiovascular diseases, we wanted to know the direct effects of IDO in obesity," explains author Soraya Taleb.

In wild-type mice, the authors showed that IDO activity in the plasma, epididymal white adipose tissue, brown adipose tissue and soleus muscle increased in those receiving a high-fat diet (HFD) compared with those receiving a normal chow diet (NCD). Ido $1^{-/-}$mice fed a NCD had a similar phenotype to the wild-type mice on this diet. However, knockout mice fed a HFD had a similar weight curve to the wild-type and knockout mice fed a NCD. These HFD-fed knockout mice also had a lower fat mass than wild-type mice on a HFD, as well as lower liver weights, less lipid accumulation in the liver and less macrophage infiltration in the liver. These findings suggest that knockout of IDO protects against the negative effects of a HFD, including weight gain and liver steatosis.
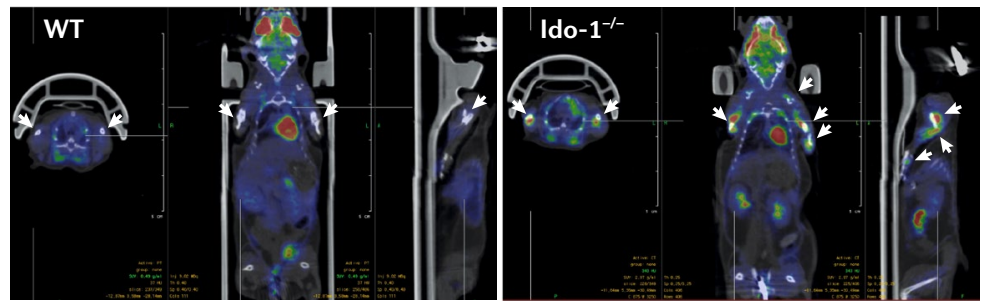

Representative fluorodeoxyglucose (FDG)-PET images (left) and quantification (right) of tissue FDG uptake in wild-type and $I d o 1^{-1-}$ mice fed with a high-fat diet for 14 weeks. White arrows show the FDG uptake in muscle. Reproduced from Laurans, L. et al. Nat. Med. https://doi.org/10.1038/s41591-018-0060-4, Springer Nature Limited. 